\title{
Designing Lentiviral Gene Vectors
}

\author{
Oleg E. Tolmachov ${ }^{1}$, Tanya Tolmachova ${ }^{2}$ and Faisal A. Al-Allaf ${ }^{3}$ \\ ${ }^{1}$ Cardiovascular Science, National Heart and Lung Institute, \\ Faculty of Medicine, Imperial College London, London, \\ ${ }^{2}$ Molecular Medicine, National Heart and Lung Institute, \\ Faculty of Medicine, Imperial College London, London, \\ ${ }^{3}$ Department of Medical Genetics, Faculty of Medicine, \\ Umm Al-Qura University, Makkah, \\ ${ }_{1,2} \mathrm{UK}$ \\ ${ }^{3}$ Saudi Arabia
}

\section{Introduction}

Gene therapy relies on the delivery of therapeutic genes into patients' cells. The microdevices used to reach the cells and to transfer the gene payload are called gene vectors. Viral packaging machinery is often utilized to generate the particles transporting the cargo genes. Lentiviruses, a subgroup of retroviruses, are highly suitable for remodeling into gene transfer vectors because they offer the stability of transgene expression, the ability to reach the nuclei of the therapeutically important non-dividing cells and are known to have a low immunogenic profile. Well studied members of the lentiviruses include human immunodeficiency viruses 1 and 2 (HIV-1 and HIV-2), feline immunodeficiency virus (FIV) and equine infectious anemia virus (EIAV).

It is important not to confuse "gene delivery vectors" and "gene cloning vectors". While the former are microparticles delivering genes, the latter are replicating vehicles for the amplification of nucleic acid sequences. "Gene delivery vectors" and "gene cloning vectors" coincide when the naked DNA of replicating bacterial plasmids or replication competent viruses is used for gene delivery into cells. Viral gene delivery vectors are normally nonreplicating and should correctly be referred to as "viral vectors", not "viruses". Particles of viral vectors can be referred to as "virions" or "transducing particles", because viral gene transfer is traditionally described as "transduction". Replication deficient viral gene vector particles are similar to "defective interfering particles", that is, faulty non-self-viable virions arising during natural viral infections and competing with non-defective virions, which were described in virology literature many years ago.

Native lentiviral envelope proteins, which determine the cell range of viral infectivity (tropism) and mediate the fusion of viral and cellular membranes, are always composed from two non-covalently attached subunits, one of which (e.g. gp41 glycoprotein in HIV-1) is membrane-embedded and the other is an external subunit (e.g. gp120 glycoprotein in HIV-1). This arrangement makes lentiviruses notoriously unstable because of their tendency to shed the external subunit of the envelope protein. As the virion's stability is a pre-requisite for the effective purification and concentration of viral vector preparations, in 
lentiviral gene vectors native lentiviral envelope proteins are routinely replaced with stable heterologous viral envelope glycoproteins, most commonly G-protein of Vesicular Stomatitis Virus (VSV) or, alternatively, G protein of Rabies Virus. Both VSV and Rabies Virus belong to the family of rhabdoviruses. VSV-G protein has a broad tropism towards lipid membranes while rabies $G$ has a distinct tropism to neural cells. The interchangeable use of envelope proteins by viruses belonging to different groups is very common and is called "pseudotyping". Lentiviral vectors can be pseudotyped with many other proteins, including artificially designed proteins, in order to improve infectivity for a particular cell type or, alternatively, to restrict the viral tropism. The rules of efficient pseudotyping are not yet completely clear, but one of the obvious requirements is the ability of the "cytoplasmic tails" of the membrane-embedded putative envelope proteins to fit into the available space between the viral capsid and the lipid envelope of the virion. Sometimes lentiviral vectors are pseudotyped by a cocktail of various viral, cellular or artificial membrane proteins. The structure of the lentiviral gene vector virion is presented in Figure 1.

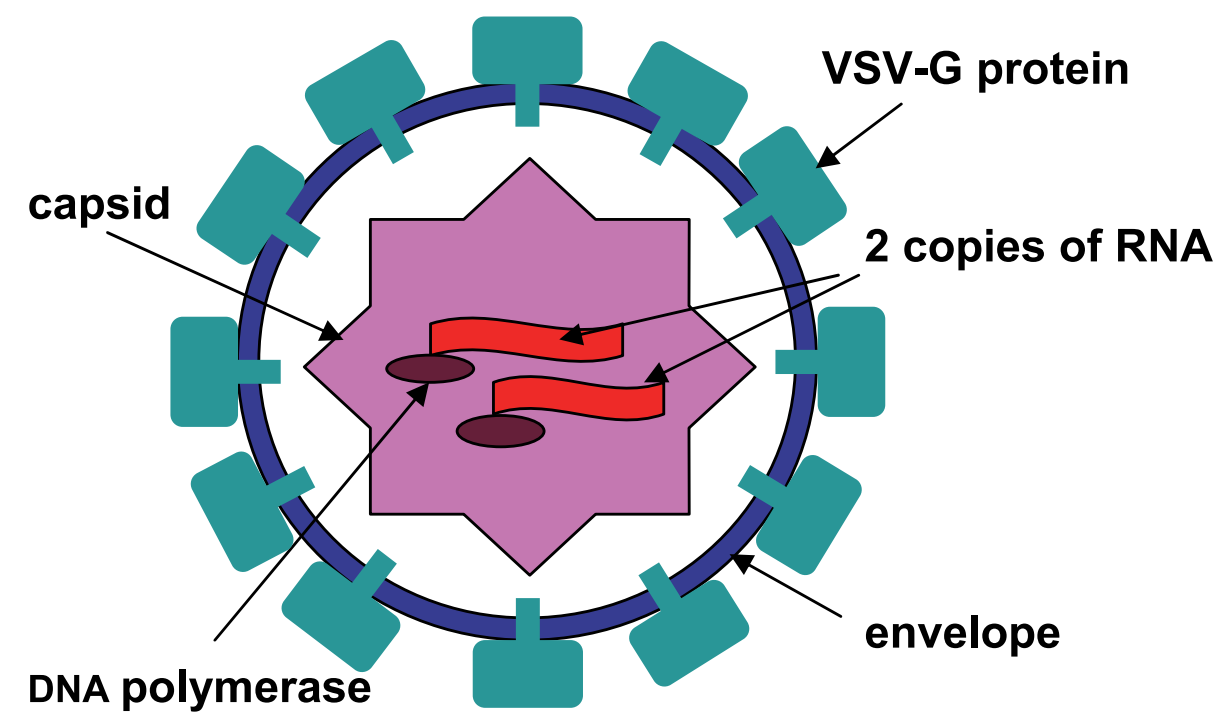

Fig. 1. A typical lentiviral vector particle

Lentiviral vectors are a promising tool for both in vivo and ex vivo gene therapy (Srinivasakumar 2001; Maier, von Kalle et al. 2010). These vectors can be used for therapeutic strategies relying on both transgene expression and gene correction (Al-Allaf, Coutelle et al. 2010). In addition, lentiviral gene vectors are extensively used in basic biomedical research to deliver genes for the expression of proteins and RNAs, e.g. shRNAs for gene knockdown. Lentiviral gene vectors are also being investigated as vaccines for immunization.

In some situations the use of classical lentiviral vectors can be compounded by the following problems: 1) insertional mutagenesis with a potential for malignant transformation; 2) relative difficulty of obtaining high titre concentrated viral vector preparations and the related problem of transduction inhibitors in the viral preparations; 3) toxicity of the commonly used VSV-G envelope protein at high multiplicity of infection; 4) limited insert 
capacity because of the RNA size packaging constraints specified by the lentiviral Gag complex.

Some of the above problems can be overcome. For example, contamination of lentiviral vector preparations with some inhibitors of infection can be avoided by the viral preparation concentration and purification by chromatography methods, which can be sufficiently gentle in comparison to ultracentrifugation based methods, which often result in a substantial fraction of the viral vector particles becoming inactivated. The reduction of viral vector losses during concentration is important, in particular because defective virions can compete with the functional virions and inhibit transduction (Geraerts, Willems et al. 2006). Perhaps the most dramatic improvement of the lentiviral vectors was the introduction of non-integrating lentiviral vectors, which do not cause genomic mutations arising via the random genomic integration of the classical lentiviral vectors (Section 5 of this review).

Lentiviral vectors can be assembled through transient co-transfection of the lentiviral vector backbone plasmid with helper plasmids expressing viral packaging functions or by stably transfected packaging cell lines. The first production method is currently a preferred choice because of its greater flexibility. A small undesirable possibility of reconstitution of the replication competent virus in the cells used for the lentiviral vector packaging can compromise safety. So, the chances of formation of a complete viral genome are customarily reduced by splitting genes for helper packaging functions between separate plasmids. For example, a common three-plasmid transient co-transfection packaging system employs a helper plasmid with genes for the structural Gag polyprotein, the catalytic GagPol polyprotein and the accessory HIV-1 Rev and Tat proteins (e.g. psPAX2) plus a helper plasmid for an envelope protein (e.g. VSV-G plasmid pMD2G). In a four-plasmid packaging system the genes for the Gag and GagPol functions on the one hand and the Rev function on the other hand are split between two different helper plasmids (with the Tat function missing altogether). Universally used cell lines for the packaging of lentiviral vectors in transient co-transfection are HEK293 cell line and its derivatives. HEK293 cells were produced by selecting an individual immortalised clone among a mixed population of human embryonic kidney cells transformed with DNA fragments of adenovirus type 5 . HEK293 cells can be efficiently transfected by the calcium phosphate method, protocols involving cationic lipids (e.g. Fugene 6 and Fugene HD) or electroporation. Contamination of plasmid DNA with co-purifying bacterial lypopolysaccharides should be avoided, as these endotoxins can substantially reduce the efficiency of transfection. HEK293T cells, which were derived from HEK293 cells and stably express large Simian Virus 40 (SV40) large T-antigen, are purported to generate retroviral and lentiviral vector preparations with particularly high titres. Thus, at present HEK293T and closely related HEK293FT cells are predominantly used for lentiviral vector production via transient co-transfection of the backbone vector plasmid and the helper plasmids. The abundance of lentiviral vector genomic RNA and levels of expression of VSV-G protein, Gag and GagPol polyproteins plus the accessory protein Rev in the packaging cells are of paramount importance for the resultant lentiviral vector titre. The ratio of a lentiviral backbone vector plasmid DNA and non-vector helper packaging plasmids is used to regulate the relative amount of genomic RNA and packaging proteins. In part, the optimal balance depends on the known toxicity of the excess VSV-G protein to mammalian cells. Clontech-Takara and ThermoFisherScientificOpenBiosystems offer mixtures of plasmids with tetracycline-inducible expression of the trans-acting packaging functions. As an alternative to plasmids, adenoviral vectors (Kuate, Stefanou et al. 2004) or baculoviral vectors (Lesch, Laitinen et al. 2011) can be used to deliver 
DNA cassettes for the transient expression of lentiviral packaging proteins and the lentiviral vector backbone modules. Clearly, this method can broaden the range of cell lines suitable for lentiviral packaging, as receptors for adenoviruses are commonly expressed by different types of cells.

The process of cell transduction by a viral vector follows the infection pathway of the cognate virus. There are several parameters characterizing the transduction of cells with a viral vector. Firstly, efficiency of transduction, defined as percentage of transduced cells out of all cells treated with a viral vector preparation, is a common and readily-obtained readout quantity in transduction experiments. The second commonly used parameter is endpoint titre, which is the number of "transduction units" per volume of the viral vector preparation with the number of transduced cells (corresponding to the "transduction units") estimated when one of the highest dilutions of the viral preparation is used to infect the cells. The third important parameter describing transduction by a viral vector is the multiplicity of infection, that is, the average number of functional transducing particles infecting one cell. The infection of a cell by a lentiviral vector normally results in the establishment of one or several copies of the corresponding provirus. The efficiency of the transduction of the cell population by a retroviral vector, which is a function of the multiplicity of infection, correlates with a number of proviruses in the genome of the infected cell (Kustikova, Wahlers et al. 2003). Thus, the obtained transgene copy number reflects the multiplicity of infection. Expression of retroviral envelope proteins is known to block superinfection (i.e. extra-infection by the same virus) of the infected cells through the depletion of the cognate cellular receptor molecules during their intracellular transport. This mechanism, though, does not apply to VSV-G protein pseudotyped lentiviral vectors, which neither have protein cellular receptors, nor code for an envelope protein under typical circumstances. Therefore, it is possible to increase the number of the lentiviral vector proviruses and, hence, transgene copy number by repeated rounds of superinfection.

As an alternative to gene delivery with packaged lentiviral vectors, the cargo genes within lentiviral vector DNA sequences can be transferred in situ "on the back" of other viral gene vectors, such as adenoviral or herpes virus based vectors. These, "dual-viral systems", e.g. adenovirus-retrovirus hybrid vectors, can deliver attenuated lentiviral vectors competent for replication and packaging in order to achieve "a booster effect" due to lentiviral vector spread in situ (Dalba, Bellier et al. 2007). In addition, such delivery systems allow for the exploitation of the cellular tropism of some well-established non-lentiviral vectors, particularly vectors based on non-enveloped viruses which lack membrane-imbedded antireceptors suitable for pseudotyping (Soifer, Higo et al. 2002).

The stable maintenance of lentiviral vectors makes them a highly suitable tool for various cell-fate mapping studies and also as sensors of cell differentiation. Tracing cells with a specific differentiation status is important in biomedical research, e.g. in stem cell studies (Gallo, Grimaldi et al. 2008). The packaging size constraints make it a challenge to avoid cross-talk between various elements within lentiviral vectors and, therefore, require the thoughtful design of lentiviral vectors with tissue specific transgene expression (Hager, Frame et al. 2008).

\section{Elements of lentiviral vector backbone plasmids}

The lentiviral vector genome has the size of about $10 \mathrm{~kb}$ and can be conveniently amplified by cloning its complete or partial DNA copy in the bacterial plasmid cloning vectors. Such 
lentiviral vector backbone plasmids are similar to other vector plasmids for mammalian gene expression, which were recently reviewed (Tolmachov 2009). The immediate purpose of the lentiviral vector backbone plasmid is to serve as a template for the transcription generating viral vector genomic RNA, which can be packaged into the lentiviral vector particles. The bacterial portion of the vector plasmid is not transcribed and, therefore, is not included in the genomic RNA of the viral vector. Thus, a typical lentiviral vector backbone plasmid consists of a bacterial plasmid portion, lentiviral elements required for viral vector RNA packaging and intracellular transport, a marker gene and/or a cargo gene and elements for their regulation, optional chromatin-control elements and sites for convenient plasmid DNA re-engineering (Figure 2).

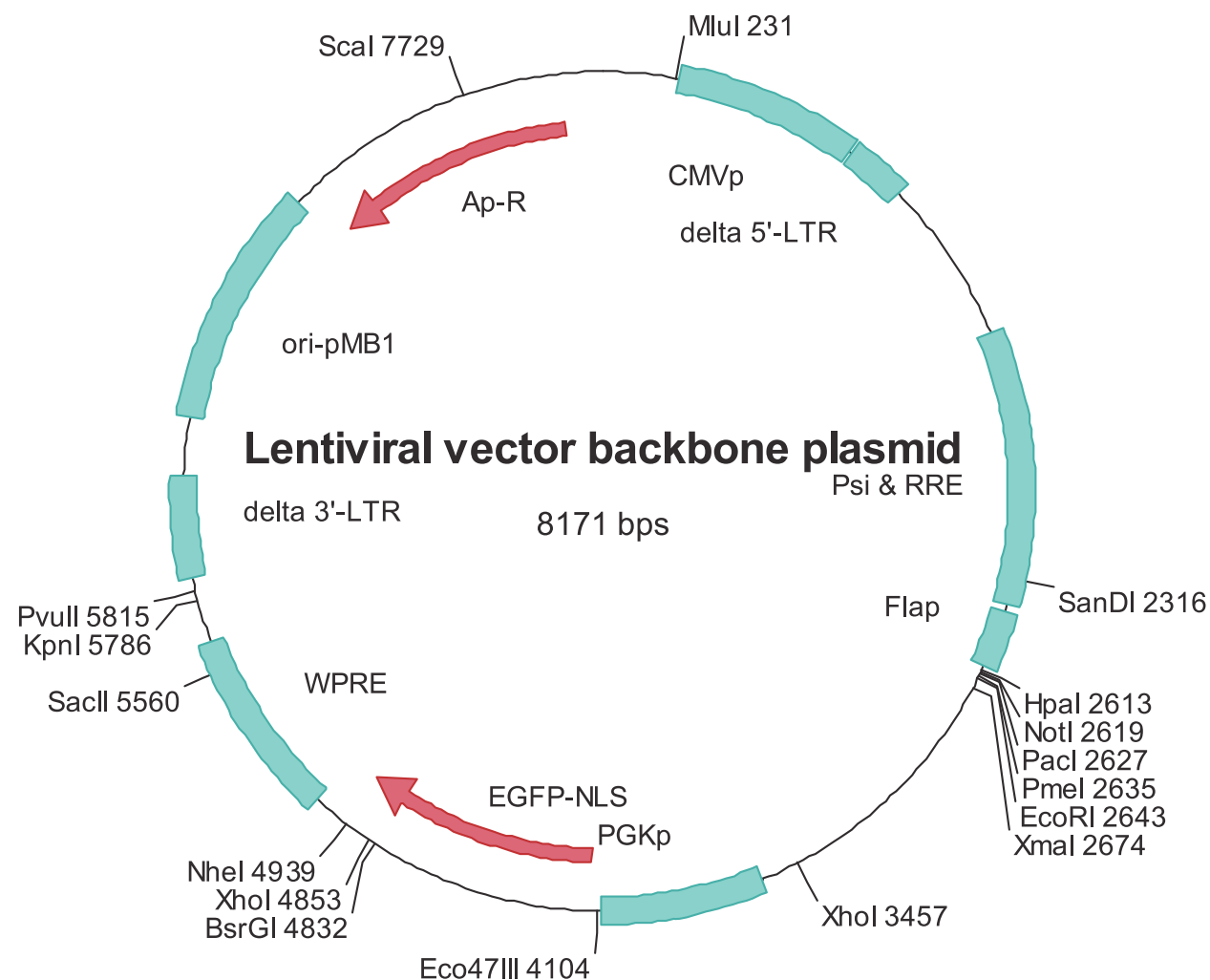

CMVp - the immediate early CMV promoter; delta 5'-LTR and delta 3'-LTR - the long terminal repeats with some deletions; Psi \& RRE - the packaging sequence $\psi$ and the Rev Response Element (RRE); PGKp - the mouse phosphoglycerol kinase promoter; EGFP-NLS - the gene for nuclear targeted enhanced GFP protein; ori-pMB1 - the multicopy origin of replication originating from the wild type plasmid pMB1; Ap-R - the ampicillin resistance marker, the gene for $\beta$-lactamase.

Fig. 2. A typical lentiviral vector backbone plasmid

Evolution has shaped both bacterial plasmid and viral genomes as mosaic assemblies, which can be disassembled into individual functional units that can then be assembled back into new chimeras. Thus, the genomes of lentiviral vectors consist of a number of genetic elements, of 
which lentiviral elements per se are only a fraction (often less than $30 \%$ of the vector genome). Lentiviral sequences essential for lentiviral gene vectors are Long Terminal Repeats (LTRs, which can be complete or partially deleted) for proviral integration and the packaging $\psi$ sequence within the genome region proximal to the $5^{\prime}$-LTR. A viral life cycle involving tight nucleic acid packaging into protein shells (capsids) requires compactness from the viral genetic elements. Thus, terse genetic elements from various unrelated viruses are particularly suitable for employment within lentiviral vector constructs. Various cargo genes can be delivered with lentiviral vectors. Because of the lentiviral packaging size constraints, the shorter, cDNAbased, versions of the genes are normally preferable. As the space within the lentiviral capsid is limited, one would only include an intron within a lentiviral vector born cargo gene if it provides an important regulatory function for the gene's expression.

Other regulatory genetic elements within lentiviral vectors include viral or cellular promoters and enhancers, splicing control elements if an intron is included, RNA stabilizing components like Woodchuck hepatitis virus Post-transcriptional Regulatory Element (WPRE) and, with a pinch of salt, polyadenylation signals (see discussion in Section 2.4).

Thus, the lentiviral vector backbone plasmid normally includes a bacterial plasmid segment, cis-elements of the lentiviral packaging machinery and an elective strong promoter to drive the synthesis of lentiviral vector's genomic RNA, cargo genes (e.g. marker genes, therapeutic genes) and genetic elements for their control. Optionally, lentiviral vector backbone plasmids can also contain sites for efficient transport of vector RNA from nucleus, for episomal maintenance of proviruses, site-specific genomic integration and also sites alleviating plasmid manipulation, e.g. polylinkers with restriction enzyme recognition sites.

\subsection{Bacterial plasmid segment}

Lentiviral vector backbone plasmids are propagated in bacteria and hence need to contain a bacterial origin of replication to drive the DNA amplification. Plasmid origins of replication are traditionally classified into a "stringent control of the plasmid copy number" group and a "relaxed control of the plasmid copy number" group. High number of plasmid copies per cell, which is typical for the "relaxed control" group, insures the high yield of the plasmid DNA. However a high plasmid copy number can place substantial stress onto the host cells, thereby decreasing their growth rate and, thus, resulting in occasional accumulation of cells with the shortened, "deletion", plasmid mutants or cells lacking the plasmid altogether. In contrast, replicons from a "stringent control" group have a lower number of plasmid copies per cell and hence comparatively small plasmid DNA yields, however plasmids driven by these replicons only rarely place a selective disadvantage onto their host cells. In addition, stable maintenance of the stringent control replicons is insured by their "partition" functions, which are responsible for the faithful distribution of plasmids between daughter cells after cell divisions. Classic relaxed control replicons are derived from plasmids ColE1 and pMB1, while typical stringent control replicons originate from plasmids RSF1010, pSC101, F-factor, and bacteriophage P1. A particularly convenient replication system, which is used in the lentiviral vector backbone plasmids, is borrowed from the plasmid R6K. This plasmid has several origins of replication, all dependent on the $\Pi$-protein encoded by the plasmid's pir gene. Thus, a lentiviral backbone plasmid with R6K $\gamma$ replicon can replicate only in the bacterial strains expressing the $\Pi$-protein. The copy number of ori-R6K $\gamma$-driven plasmids can be increased substantially if the plasmid is transferred to an array of strains expressing various "copy-up" mutant versions of the П-protein, thus giving the flexibility to choose the optimal copy number for a particular lentiviral backbone plasmid. 
To select bacterial tranformants during plasmid modifications and to prevent plasmid loss using selective pressure for plasmid-containing cells, the bacterial plasmid segment should include a suitable selection marker. The standard solution is to employ an antibiotic selection gene such as the ampicillin resistance gene for $\beta$-lactamase or the chloramphenicol resistance gene for chloramphenicol acetyl transferase. Large scale preparations of plasmid DNA with antibiotic resistance genes might lead to the undesirable escape of these genes into environment. Thus, genetic systems, which rely on a short RNA-expressing gene as a plasmid selection marker, can be contrived (Luke, Carnes et al. 2009).

Regrettably, it is relatively common for lentiviral vector backbone plasmids to suffer from structural and maintenance instability in bacteria. There are several common factors contributing to this instability. Lentiviral vector backbone plasmids are necessarily greater than $9 \mathrm{~kb}$ in size; if their replication is driven by a replication origin with "relaxed control" of the plasmid copy number, spontaneously arising deletion mutants have a propensity to replicate faster and, as a result, tend to establish dominance in the mixed plasmid population. Plasmid deletions can occur through homologous or illegitimate recombination between inverted repeats of the lentiviral LTRs. A mosaic of eukaryotic sequences within the lentiviral vector backbone plasmid might contain cryptic bacterial promoters, which could be able to drive the expression of toxic RNAs and polypeptides creating a negative selection pressure on the population of bacterial cells harbouring the desired plasmids. It should be noted that the ampicillin resistance gene coding for $\beta$ lactamase, which is often used in the commercially available lentiviral backbone plasmids as a selection marker, is poorly suited for long-term bacterial selection in liquid cultures. This is because wild type $\beta$-lactamase is secreted in Escherichia coli and can destroy ampicillin before the bacterial culture is fully grown. Thus, if a particular lentiviral backbone plasmid would have a maintenance problem, the overgrowth of plasmidless cells in liquid culture would not be stopped by ampicillin. In such a situation, the use of alternative non-ampicillin-based selection marker is recommended. Alternatively, as much bacterial cultivation as possible should be performed on a solid agar medium containing ampicillin, where the spread of ampicillin-destroying $\beta$-lactamase is slower. For example, a seeding stock for a liquid plasmid maxi-preparation culture can be grown from an individual colony on agar plates with ampicillin instead of a liquid overnight culture. Non-secreted, cytosolic, mutant versions of $\beta$-lactamase were described and can be used for efficient selection of bacteria harbouring maintenance-compromised lentiviral vector backbone plasmids.

\subsection{Cis-acting elements of the lentiviral molecular machinery}

The standard purpose of lentiviral vector backbone plasmids is to provide a template for the synthesis of lentiviral vector genomic RNA, which can be successfully packaged into lentiviral vector virions, reverse transcribed and integrated within the cellular genome. The $\Psi$-sequence close to the $5^{\prime}$-LTR is strictly required for the packaging of RNA by the Gag polyprotein. A substantial portion of the sequences within the LTRs of the lentiviral genome is required for chromosomal integration. However, some sequences within the LTRs can be removed without reduction in the integration efficiency. Wild-type retroviral and lentiviral genomes contain a promoter within their 5'-LTR to drive expression of genomic RNA. The promoter sequences can be deleted from the 5'-LTR DNA segment in the lentiviral vector backbone plasmids and a strong constitutive promoter capable of directing synthesis of the vector genomic RNA, e.g. 
immediate early CMV promoter, can be placed externally to the bracket of the lentiviral sequences within the plasmids. As the accessory lentiviral protein Tat is required for the activation of the promoter within the wild type $5^{\prime}$-LTR of HIV-1, the use of an external promoter for genomic RNA synthesis allows exclusion of the Tat gene from the packaging system with the consequent reduction in the probability for the re-constitution of the replication competent virus and the corresponding improvement in safety (Dull, Zufferey et al. 1998). A substantial fraction of the currently used lentiviral vectors are "self-inactivating" (SIN) due to a deletion within their 3'-LTR. Indeed, as the DNA-copy of the 5'-LTR of the vector provirus is always synthesized by reverse transcription from the template of the $3^{\prime}$-LTR, proviruses of the SIN vectors lack sequences required for the re-constitution of the lentiviral promoter within the $5^{\prime}$-LTR segment of the vector provirus and the synthesis of the lentiviral vector genomic RNA from the provirus template. Thus, the SIN-vectors cannot be redistributed from the target cells, which is beneficial for their safety profile.

Lentiviruses possess molecular machinery to enter into the nuclei of postmitotic, nondividing cells with an intact nuclear envelope. Lentiviral nuclear-penetration apparatus is in part dependent on the triple-stranded structure created during reverse transcription of the lentiviral genomic RNA, "the central DNA flap" (Riviere, Darlix et al. 2010). Central Polypurine Tract (cPPT) and Central Termination Sequence (CTS) are required for the formation of "the flap". Therefore, DNA sequences for CPPT and CTS need to be present in the plasmid template of the nuclear penetrating lentiviral vectors.

Rev protein of HIV-1 is known to increase lentiviral vector titres by promoting the export of genomic RNA from the nucleus. This export depends on the Rev Response Element (RRE) sequence within genomic RNA. Therefore, the RRE sequence is a desirable building block for inclusion into the lentiviral vector backbone plasmids.

\subsection{Marker genes for mammalian cells}

The concentration of lentiviral vector particles can be assessed by their ability to transduce cells and by physical measurements estimating the number of virions in a volume. A substantial number of virions in lentiviral preparations can be infection defective, so transduction data are vital for the evaluation of the titre of functional lentiviral vector particles. Convenient markers for the assessment of efficiency of transduction and for the fate mapping of transduced cells include genes for fluorescent proteins, drug resistance proteins, luciferase and cell surface antigens. More practical markers are easily detected in live cells, while others require fixation of the live material. Enzyme coding genes, such as the lacZ gene for bacterial $\beta$ galactosidase, can be used as transduction markers, but lost some of their initial popularity because of the need to fix and, thus, to kill the transduced cells before colour reaction with enzymatic substrates. In addition, the lac $Z$ gene is large (3075 bp), and occupies a substantial portion of the permissible payload size within lentiviral vectors. Similarly, detection of intracellular proteins by immunostaining requires cells to be killed by permeabilization of their membranes and fixation. Thoughtful choice of the viral vector marker is important for the straightforward collection of data and their faithful interpretation. Most marker proteins are small and do not illicit a substantial immune reaction in vivo. It is important to plan a convenient intracellular localization of the marker proteins as this can affect their activity and can minimize background signal. Genetic determinants used to direct proteins to a specific subcellular compartment include nuclear localization signals (NLS), chromosome binding moieties (e.g. histon $\mathrm{H} 2 \mathrm{~B}$ ) and domains for plasma membrane association, endoplasmic 
reticulum retention, targeting to mitochondria, peroxisomes, Golgi-apparatus, actin filaments and microtubules. Fused signal sequences or protein domains must not interfere with the marker protein multimer formation if the quaternal protein structure is required for its activity. Drug resistance (normally antibiotic resistance) genes are especially suitable for tasks requiring the establishment of stably transduced cell clones originating from individual transduced cells. The choice of a particular drug resistance gene can be affected by its size. The gene for blasticidin resistance, $b s r$, is $423 \mathrm{bp}$ long and is particularly compact. In contrast, the gene for hygromycin resistance is $1038 \mathrm{bp}$ long, and, therefore, is a less attractive choice if the available space within the lentiviral vector is limited. Different drugs cause cell death with dissimilar rates and their minimal inhibitory concentrations vary for different types of cells. The number of surviving transduced cell clones is typically higher at lower drug concentrations. Other frequently used antibiotic resistance markers include genes for resistance to zeocin, puromycin and neomycin analogue G418. Drug sensitivity genes, such as HSV thymidine kinase (TK), can also be delivered by lentiviral vectors. These genes are highly instrumental for cell suicide systems in cancer gene therapy, but are difficult to use as transduction markers.

Fluorescent proteins are remarkably convenient as gene transfer markers because they can be detected both in live and fixed cells. The largest family of fluorescent proteins originates from the green fluorescent protein (GFP) of jellyfish Aequoria victoria. Systematic protein engineering efforts produced mutant derivatives of GFP with increased brightness and widely different excitation and emission spectra (e.g. blue, cyan and yellow fluorescent proteins). Similarly, dsRed protein of coral Discosoma sp. was used to create a family of proteins including the orange fluorescent protein mOrange and the far-red fluorescent protein E2-Crimson. A number of specialized versions of fluorescent proteins were obtained, e.g. reduced half-life GFP for the collection of dynamic gene expression data. Fluorescently activated cell sorting (FACS) is the method of choice both for analytical analysis of the transduced fluorescent cells and for their preparative isolation. Fluorescencebased microscopy is another convenient and informative method; however, 3-D imaging of fluorescent material is complicated by the extinction of both excitation and emission light in the thick slices. Still, the image data collected from thin slices can be assembled into 3-D images using computational algorithms. Computational algorithms can be also applied for "image de-convolution" in order to achieve some improvement of image quality using fluorescence data obtained directly on the thick specimens.

The titre of a lentiviral vector depend to a large degree on the type of the marker used for analysis. Indeed, the titre determined by detecting the fluorescent transduction markers is usually higher than the titre obtained by counting drug resistant cell clones.

Genes for luminescence proteins can be transferred by lentiviral vectors and used as cell tracers (Reumers, Deroose et al. 2008). Luciferases from North American firefly Photinus pyralis and sea pansy Renilla reniformis are commonly used. The two luciferases use different substrates for light production, this can be exploited to create internal control for luminescence in "dual luciferase reporter assays" used, e.g., for promoter strength testing or shRNA/siRNA analysis. The "baseline" luciferase marker (normally Renilla luciferase) can be delivered for expression on a separate vector or on the same vector. A version of firefly luciferase with enhanced expression in mammalian cells (luc2) was developed by Promega by codon optimization (as discussed in Section 2.4) and elimination of predicted transcription factor binding sites within the reporter gene sequence. On the basis of luc2, 
Promega developed luciferases with a higher rate of degradation such as luc2P (containing PEST protein degradation signal ("degron") on the C-terminus or luc2CP (containing CL1 and PEST protein degrons on the C-terminus). PEST is a 40 amino acid sequence present in the C-terminal region of mouse ornithine decarboxylase. CL1 is a 16 amino acid degron from yeast. This signal was also optimized for expression in mammalian cells. Versions of luciferase with the degradation signals improve responsiveness to factors enhancing or inhibiting luciferase expression. Other specialized versions of luciferases are available. For example, secreted firefly luciferase is convenient to measure the luciferase activity of live cells in tissue culture. Renilla luciferase-neo ${ }^{\mathrm{R}}$ fusion protein was generated and can be used for both cell clone selection and as an internal luminescence control. In vivo "whole animal" detection of luminescence is a common approach with no excitation light being required and with the efficient amplification of emitted photons. However, low spatial resolution limits due to light dispersion in the animal restrict the expediency of this approach.

Surface antigen markers, e.g. extracellular domain of CD4, truncated low-affinity nerve growth factor (LNGFR) or truncated mouse MHC class I molecule $\mathrm{H}-2 \mathrm{~K}^{\mathrm{k}}$ can be used as cell markers for antibody-mediated conjugation with paramagnetic particles, which can be used for both magnetic cell sorting and magnetic resonance imaging (So, Hotee et al. 2005). The intracellular domains of these proteins are removed to avoid signal transduction and the extracellular domains are supplied with GPI-lipidation signal for plasma-membrane anchoring (as in Miltenyi Biotec MAC Select ${ }^{\mathrm{TM}}$ system). Background signal during wholeanimal imaging can be avoided by using species-specific monoclonal antibodies for the cell surface marker proteins in a heterologous host organism.

\subsection{Genetic elements for controlling the expression of marker genes and cargo genes in lentiviral vectors}

In general, cis-acting elements strictly required for gene expression in eukaryotes are a minimal promoter and a transcription terminator. Genes coding for proteins (cistrons) also necessarily contain: 1) the translation start codon ATG with the surrounding Kozak consensus sequence controlling translation initiation; 2) the protein coding sequence; 3) the stop codon. Protein-encoding genes are transcribed by eukaryotic Polymerase II from Polymerase II specific promoters. The sequence of the Polymerase II promoters is variable; however, "TATAbox" with the consensus sequence 5'-TATAAAA-3' is normally found 20-25 bp upstream of the transcription start site. In addition, minimal Polymerase II promoters often contain "CATbox" with the consensus sequence 5'-GGCCAATCT-3' at the position -70 relative to the transcription start site. The Kozak consensus sequence is important for expression of proteincoding transgenes born on lentiviral vectors. The DNA template for one of the strong versions of the Kozak sequence can be written down as 5'-gccaccATGgcg-3'.

The Polymerase II transcripts, e.g. mRNAs, are nearly always modified by the addition of 7methylguanosine (the "cap") to their $5^{\prime}$-ends and the addition of the homopolymeric tail of adenosine nucleotides to their $3^{\prime}$-ends. Polyadenylation of eukaryotic mRNAs is important for their protection from exonucleolytic attack and for their export out of nucleus. It also serves as a means of transcription termination. For mRNA to be polyadenylated, it should contain a specific signal sequence downstream of its polypeptide coding sequence. In general, a DNA sequence for the functional "polyadenylation signal" (pA signal), which is suitable for insertion into lentiviral vector backbone plasmids, is several hundred nucleotides long and is borrowed from a mammalian gene or a viral genome. Commonly 
used pA signal sites are taken from the rabbit $\beta$-globin gene, human growth hormone gene and human herpes virus (HSV) thymidine kinase (TK) gene. The sequence 5'-AAUAAA-3' located 10-30 nucleotides upstream of the cleavage site is highly conserved but is not strictly required for the polyadenylation of mammalian mRNAs. A very important feature of the lentiviral vector backbone is the pA signal in its 3'-LTR. This signal functions as a terminator for wild type lentiviral RNAs including complete lentiviral genomic RNA. As the 3'-LTR sequence itself is strictly required for chromosomal integration in lentiviruses, any foreign pA signals which can cause premature termination of the genomic RNA are bound to reduce the amount of transductionally active genomic RNA, and, therefore, to diminish drastically the titre of the functional lentiviral vector particles (Hager, Frame et al. 2008). Thus, foreign pA signals within lentiviral vectors should be either avoided altogether, or weakened, or positioned to terminate the transcription of the anti-genomic DNA strand only. With this challenge in view, it should be noted that while most pA signal sites act unidirectionally, a pA signal borrowed from SV40 viral genome is known to terminate RNA and to promote polyadenylation irrespective of transcription direction. A brief look at the transcription map of SV40 can explain this fluke. Indeed, two opposing transcription waves of SV40 meet and terminate at its polyadenylation signal site, which is, therefore, an overlap of two opposing polyadenylation signals.

Natural Polymerase II promoters are nearly always supplemented with one or several "enhancers", that is, genetic elements, which up-regulate the activity of the promoter through binding to specific nuclear proteins, so-called "transcription factors". Size limitations of the lentiviral payload and insufficiently precise enhancer localization data restrict the use of enhancers in the lentiviral vectors. However, small enhancer elements can still be used where, for example, tissue-specific or inducible transgene expression is desired. A typical eukaryotic protein-coding gene is a patchwork of coding exons and non-coding introns, so that the translation-grade mRNA is produced by the splicing of the primary transcript. The result of the splicing is the establishment of the phosphodiester bond between the AG dinucleotide at the $3^{\prime}$-end of the preceding exon and the $\mathrm{G}$ nucleotide at the 5 '-end of the subsequent exon within the transcript (Hiller and Platzer 2008). The interlacing sequences, the introns, are flanked with a "splice-donor" consensus sequence 5'-GTRAGT-3" at their $5^{\prime}$-ends and a "splice-acceptor" sequence 5-YAG-3" at their $3^{\prime}$-ends (where $\mathrm{R}$ is A or $\mathrm{G}, \mathrm{Y}$ is $\mathrm{C}$ or $\mathrm{T}$ ). In addition, introns contain a "branch point" $5^{\prime}$-YTRAY-3" and a polypyrimidine tract, which are functionally important for successful splicing. As the payload space within the lentiviral vectors is limited, the standard practice is to include the genes in their complementary DNA (cDNA) form, that is, as spliced versions without introns. The introns, however, are not entirely inert genetically and occasionally take part in the regulation of gene expression. In such situations, the inclusion of small regulatory introns within lentiviral vectors can be considered (Le Hir, Nott et al. 2003).

Coding sequences delivered by lentiviral vectors are often derived from non-mammalian organisms where the translation machinery is adapted to a non-mammalian profile of codon frequencies. Unusual codons, also called "hungry codons", can cause undesirable pauses during translation and reduce the efficiency of gene expression. Therefore, the optimization of codon frequencies for the genes, which are born on the lentiviral vectors, is often advantageous. If the frequencies in a coding sequence are adapted to the human codon usage profile, the sequence is said to be "humanized" (Zeeberg 2002; Burgess-Brown, Sharma et al. 2008). 
The stability of genomic RNA of lentiviral vectors is crucial for attaining high lentiviral vector titres and stability of the lentiviral vector encoded mRNAs is important for efficient transgene expression. It was discovered that an element from Woodchuck Hepatitis Virus (WHV) genome can operate at a post-transcriptional level to improve transgene expression. The sequence, called WHV Post-transcriptional Regulatory Element (WPRE) is thought to act by expediting the export of RNA from the nucleus with the concomitant reduction of the intra-nuclear RNA degradation. WPRE and a similar element from Human Hepatitis Virus (HBV) are extensively used in lentiviral vectors, where they are normally positioned immediately upstream from the 3'-LTR, primarily because of their vector titre enhancing properties. WPRE, as defined originally, was known to cause tumours in rodents, therefore, a safer version of WPRE with deleted sequences for the WHV X protein and its promoter was generated (Schambach, Bohne et al. 2006).

Cistrons are not the only cargo genes delivered by the lentiviral vectors. Other payload genes include the genes for RNAi-exploiting "micro" RNAs (miRNAs) or their artificial analogues, "short hairpin" RNAs (shRNAs). These genes are transcribed by RNA Polymerases III or I from the corresponding promoters. The benefits of the lentiviral vectors, such as the relative stability of transgene expression and the ability to transduce postmitotic cells, considerably broaden the versatility of gene knock-down experiments with shRNAs (Rubinson, Dillon et al. 2003). A range of suitable lentiviral vectors, exploiting the high activity of hybrid miRNA-30-shRNA design, are offered by ThermoFisherScientificOpenBiosystems (Silva, Li et al. 2005).

In summary, cis-acting elements regulating gene expression act in concert and in a cell-typespecific manner. The inherent mosaicism of lentiviral genetic organization allows interchangeable use of an extensive array of genetic elements for the generation of new lentiviral vectors. However, some combinations of genetic determinants are less functional than others, so the optimization of the lentiviral vector set-up is usually required.

\subsection{Genetic elements for epigenetic maintenance of transgene expression}

It is a relatively common occurrence for transgene expression to die out both in terms of the reduction of the fraction of expressing cells and the decrease of the efficiency of expression. Integrated lentiviral proviruses are faithfully maintained in mammalian cells, so the reasons for the shutdown of transgene expression are mostly epigenetic. Malfunction of the transgene expression control elements is often blamed, indeed, the phenomenon is sometimes referred to as "promoter shut down". Certainly, different promoters have various capabilities to maintain long-term transgene expression. In particular, some promoters tend to turn off in cell populations where they are not normally active. The shutdown of transgene expression is particularly common in cell populations undergoing differentiation (Bagchi, Kumar et al. 2006). Natural chromosomal integration of lentiviruses tends to occur in transcriptionally active areas of the genome where heterochromatin and DNA methylation are unlikely to interfere with transgene expression. However, as the cells differentiate, the pattern of heterochromatization and DNA methylation changes and some of the proviruses find themselves in the transcriptionally silent areas of the genome.

There are many levels at which the longevity of transgene expression can be addressed through the lentiviral vector design, including: 1) control of the provirus amenity to methylation (e.g. purposeful exclusion of the methylation-prone CpG islands); 2) chromatin re-modeling control via cis-acting proviral elements; 3) choice of non-immunogenic 
transgene products to prevent cell elimination via immune reaction in vivo; 4) choice of a suitable tissue-specific promoter-enhancer combination; 5) achieving the high copy number of proviruses; 6) control of the provirus integration sites via the preferences of the viral integrase or via harnessing the site-specific integration systems.

In principle, the protection of proviruses from heterochromatin can be achieved with genomic insulators or other similar anti-heterochromatin elements. However, experiments with known insulators show that their effects on transgene expression from lentiviral proviruses are multi-vectorial depending on the cell context (Grandchamp, Henriot et al. 2011). Ideally, targeting proviruses to a continuously active locus (e.g. human homologue of the mouse Rosa 26 locus) can resolve the transgene expression shutdown problem. An alternative solution is to escape chromatin-remodeling events by creating episomally maintained lentiviral proviruses (Section 5).

\subsection{Elements alleviating manipulation of lentiviral vector backbone plasmids}

The fairly large size of the lentiviral vector backbone plasmids means they contain a limited number of unique sites for restriction nucleases. Thus, it is often desirable to introduce artificial clusters of suitable unique restriction sites (polylinkers) to simplify the modification of these plasmids. Alternatively, selection schemes involving site-specific recombination can be used for repetitive modifications, e.g. for marker exchange or promoter exchange. In this scenario, the introduction of suitable site-specific recombination sites into the lentiviral vector backbone plasmid is required.

As discussed in Section 2.1, lentiviral vector backbone plasmids are occasionally structurally unstable and/or poorly maintained. These plasmids are particularly vulnerable during initial establishment in bacteria. Instability of nascent recombinant plasmids can result in a practical unfeasibility of seemingly straightforward DNA cloning strategies. For example, the generation of new lentiviral vector backbone plasmids through inefficient ligation of two DNA fragments with blunt ends is normally very challenging. In such situations we recommend splitting the DNA cloning procedure into separate cloning steps, each one relying on either effective positive selection of new recombinant plasmids or on efficient ligation. For example, the insertion of additional DNA sequences into lentiviral vector backbone plasmids can take advantage of the "pop-in-pop-out" cloning strategy. In this approach, a plasmid containing the desired insert and marked with antibiotic resistance marker 1 is first fused with the lentiviral vector backbone plasmid marked with antibiotic resistance marker 2 using positive selection of the co-integrate plasmid with two antibiotics. The desired new lentiviral vector backbone plasmid is then obtained by removing the unwanted plasmid sequences from the resultant bi-replicon plasmid using restriction digestion and efficient intra-molecular ligation reaction. Clearly, this strategy for insertions into a lentiviral vector backbone plasmid requires the thoughtful placement of restriction sites conveniently accommodating both the "pop-in" step and the "pop-out" step.

\section{Engineering lentiviral vectors for the concomitant expression of several transgenes}

The genome sizes of non-defective wild type HIV-1 isolates are close to $9.5 \mathrm{~kb}$. The lentiviral packaging size constraints are dictated by the geometry of the viral capsid and are thought to be fairly permissive of the smaller than wild type genome versions, but remarkably 
intolerant of the larger than wild type variants. As lentiviral sequences required for genomic RNA packaging and chromosomal integration constitute about $2 \mathrm{~kb}$, the available gene payload space within HIV-1 based lentiviral vectors should not be much more than $7.5 \mathrm{~kb}$. Thus, the insert size capacity of the lentiviral vectors is completely appropriate for the vast majority of the monogenic applications but can present a challenge in situations where the delivery of several genes by a single vector is required. Therefore, various possible methods of gene cargo reduction have been explored.

The expression of two or more cistrons from a single promoter can be achieved by the employment of internal ribosome entry site (IRES) elements, which are normally borrowed from viral genomes (Fux, Langer et al. 2004). The IRES sequences (about $0.5 \mathrm{~kb}$ ) are inserted between the coding frames to facilitate translation of the downstream coding frames from the same transcript. IRES efficiency is not absolute and it is a common occurrence for the subsequent gene in the expression cassette to be expressed at a lower level than the preceding gene. As the length of the intercistronic inserts is an important factor in the IRES efficiency, the expression of the downstream genes can be improved by increasing or decreasing the sizes of the IRES inserts (Attal, Theron et al. 1999).

Another common method used to compress the gene expression cassettes is to produce gene products as polyproteins, that is, large polypeptides, which are split into individual proteins by proteases recognizing the appropriate amino acid sequence cleavage motifs between the protein modules. In fact, lentiviruses use the same principle themselves as the Gag polyprotein is proteolytically processed inside the viral particles to form nucleocapsid, capsid, matrix proteins and the GagPol polyprotein is processed to form additionally viral protease, integrase and reverse transcriptase. The usual proteolytic signal, $2 \mathrm{~A}$, which is used in recombinant lentiviral gene vectors, is, however, borrowed not from lentiviruses but from the Foot-and-Mouth Disease Virus belonging to the family of picornaviruses. Multiple copies of the 2A sequence can be successfully used to indicate the desired break-up of the polyproteins. The method has its limitations as protein misfolding might occur, which would require some extra chaperone support.

Quite often there is no need to separate several proteins as they can perform several functions remaining as a single polypeptide chain. In general, multifunctional fusion proteins are produced by the fusion of the coding sequences in the same translation frame. Some spacer peptides might be used to intercalate between the fused polypeptides to improve folding and to facilitate the functional activity of the individual protein domains. There are two common methods to achieve protein fusion: 1 ) the stop codon of the upstream coding unit is deleted and the start codon of the downstream coding unit is retained to obtain alternative translation starts; 2 ) both the stop codon of the upstream coding unit and the start codon of the downstream coding unit are deleted. It is important to remember that many proteins are naturally proteolytically processed and this processing can be upset by protein fusion or can interfere with the desired fusion. For example, many proteins are secreted into the lumen of the endoplasmic reticulum (ER) with the concomitant cleavage of the leader peptide by the signal peptidase. If incorporated within the downstream portion of the fusion protein, the signal peptide cleavage sequence can cause undesired fission of the fusion protein. Thus, potential problems associated with building of novel fusion proteins include protein misfolding, incorrect intracellular localization and unexpected sensitivity to a protease attack. In addition, in vivo use of fusion proteins might be impeded by unwanted immunogenicity of the newly-created epitopes. 
Mimicking wild type lentiviruses, splicing signals can be exploited to generate multiple mRNAs for different proteins from a single primary transcript (Zhu, Chung et al. 2001). However, internal splicing signals reduce the amount of the full size genomic RNA of the lentiviral vectors and, therefore, are bound to be detrimental for the vectors' titres.

On some occasions several cargo genes within one lentiviral vector can be arranged to be driven by their individual promoters. This option is discussed in the following Section 4 .

\section{Multiple transcription modules in lentiviral vectors}

The most straightforward way to arrange two transcription cassettes within the lentiviral vector is to assemble them in a tandem with the orientation coinciding with the transcription direction for the primary genomic RNA (that is, from 5'-LTR to 3'-LTR). Extreme caution is recommended in the employment of foreign pA signals in both cassettes, as they can interfere with the production of functional genomic RNA of the lentiviral vector. The production of functional genomic RNA and transcription from the two gene expression cassettes can all rely on the pA signal within $3^{\prime}$-LTR and a postranscriptional enhancer, such as WPRE, positioned immediately upstream from the $3^{\prime}$-LTR. Such tandem arrangement of two gene expression units is prone to overriding any regulatory features of the downstream promoter by the topmost upstream promoter. Thus, a regulated promoter (e.g. a tissue specific promoter) should be positioned in the upstream gene expression unit while a constitutive and ubiquitous promoter should be positioned downstream (Gallo, Grimaldi et al. 2008; Kita-Matsuo, Barcova et al. 2009). As the lentiviral promoter in the 5'LTR can override any regulatory features of the downstream promoters, versions of the lentiviral vectors with a deleted $5^{\prime}$-LTR promoter are preferable for tandem assemblies of expression cassettes, which include a regulated promoter. There are multiple situations, both in gene therapy and in general biomedical research, where an induced transgene expression is required. In particular, heat (Vilaboa and Voellmy 2006), light (Schoenenberger, Gerosa et al. 2009; Deisseroth 2011) and gas-born acetaldehyde (Weber, Rimann et al. 2004) were used for induction of gene expression in mammalian cells (Goverdhana, Puntel et al. 2005).

An alternative solution for the arrangement of two expression cassettes within a lentiviral vector genome is an assembly with the divergent orientation of the transcription. The advantage of the divergent orientation of the promoters is that it excludes any cross-talk between two gene expression units. Therefore, two highly regulated promoters can be employed in the same vector. An expression cassette positioned along the transcription of the genomic RNA can still use pA signal in 3'-LTR while a counter-genomic-transcription unit requires its own pA signal. Care should be taken to use an exclusively unidirectional $\mathrm{pA}$ signal and not to use a DNA fragment with two opposing pA signals, which can cause the disruption of the functional genomic RNA production.

Lentiviral vectors with tissue-specific promoters can be used for the long-term expression of transgenes in gene therapy and also as sensors of cell differentiation, an important task in stem-cell-based therapy. There are two principal types of genetic sensors: a "responsive" type with a real-time reaction to the cell's state and a "fate-mapping" type, which, once activated, can permanently retain the sensor state during any epigenetic changes happening while cells differentiate or react to outside stimuli. As promoters and enhancers are pivotal elements of the gene expression control, the ability of lentiviral vectors to accommodate several transcription cassettes is an important consideration in the gene vector choice. 
A flexible way to regulate gene expression is via cistron inversion relative to a promoter (Atasoy, Aponte et al. 2008; Sohal, Zhang et al. 2009). Thus, a site-specific recombinase, such as Cre-recombinase, can react with the cognate recombination sites within lentiviral vector provirus to activate or to block gene expression. Similarly, controlled excision or inversion of a transcription terminator can be used as a regulatory contrivance. The expression of some genes, e.g. cell suicide genes in cancer gene therapy, requires extra tight control, which can be achieved via a parallel or cascade arrangement of two control elements such as an inducible promoter and a cistron-inversion system (Sektas, Hasan et al. 2001). Lentiviral vectors seem to be particularly suited for the assembly of complex gene expression control systems destined for delivery to postmitotic cell populations as their packaging size constraints are less prohibitive than the packaging size constraints of competing AdenoAssociated Virus (AAV) based gene vectors.

\section{Generating non-integrating lentiviral vectors}

One of the current trends in vectorology is for viral vectors to acquire some of the advantageous features of non-viral vectors and for non-viral vectors to borrow attractive bits of the viral machinery. A clear example of that trend is the rapid coming to the fore of non-integrating lentiviral vectors, which are transducing particles still bearing a substantial resemblance to lentiviruses and yet deficient in typical for retroviruses random chromosomal integration of their proviruses (Nightingale, Hollis et al. 2006; Philippe, Sarkis et al. 2006; Yanez-Munoz, Balaggan et al. 2006; Apolonia, Waddington et al. 2007; Philpott and Thrasher 2007; Bayer, Kantor et al. 2008; Sarkis, Philippe et al. 2008; Rahim, Wong et al. 2009). Importantly, non-integrating lentiviral vectors retain the nuclear-penetrating ability, the flexibility of virion envelope engineering and capacity to package nucleic acids of the therapeutically relevant size that are otherwise characteristic of the lentiviral gene vectors (Wanisch and Yanez-Munoz 2009). The benefits of the non-integrative vectors for gene therapy arise from the absence of malignancy provoking insertional mutagenesis, which can occur because of the disruption of the tumour supressor genes or activation of expression of the oncogenes after proviral integration.

Non-integrating lentiviral vectors are prepared with modified lentiviral packaging systems, which employ a modified GagPol polyprotein containing mutations specifically inactivating the lentiviral integrase function. If a regular lentiviral vector backbone plasmid is used in the packaging procedure, the resultant vector virions can deliver transgenes to the nucleoplasm, where the transgenes can stay and be expressed until they are diluted out in cell divisions. Therefore, such vectors are suitable either for transient transduction of dividing cells or for relatively stable transduction of non-dividing cells, where the lentiviral vector genomes can persist. To achieve better maintenance of the transgenes, non-integrating lentiviral vectors have to be supplemented with either site-specific integration machinery (Lombardo, Genovese et al. 2007; Moldt, Staunstrup et al. 2008) or episomal maintenance apparatus (Wong, Argyros et al. 2009; Argyros, Wong et al. 2011).

Provirus integration can be made harmless by its targeting to a benign locus within the target cell genome (Moldt, Staunstrup et al. 2008). The lentiviral integrase itself can be engineered into a site-directed recombination enzyme by its fusion with site-specific "tethering" domains (Ferris, Wu et al. 2010). Robust and error-free site-specific integration into mammalian cells lacking pre-engineered integration sites is, however, difficult to achieve. Thus, compact episomal replicons from SV40, polyoma, papilloma viruses or 
EBNA1-Rep1 DNA segment of Epstein-Barr virus (EBV) can be used to support maintenance of non-integrating lentiviral vectors in the nucleoplasm of dividing cells. Viral replicons are often completely adequate for research use of gene vectors, however they are rarely acceptable for therapeutic applications. Indeed, expression of the large SV40 Tantigen and, hence, malignant transformation of the recipient host cells is required for SV40origin-based replication. EBNA1 expression does not result in a typical malignant transformation but can still tilt the cells towards the undesired immortalisation (Humme, Reisbach et al. 2003). Alternative benign episomal replicons are being sought; encouragingly, the scaffold/matrix attachment region (S/MAR) from the human $\beta$ interferon gene was reported to support episomal replication (Wong, Argyros et al. 2011).

\section{How to enhance the transduction of cells with lentiviral vectors}

The titre of lentiviral vector particles in the cell culture supernatant hardly ever exceeds $1 \times 10^{8} \mathrm{TU} / \mathrm{ml}$ and, depending on a particular lentiviral vector backbone, is often much lower. Therefore, the use of concentrated lentiviral vector preparations is a popular, even though relatively involved, approach to achieve high efficiency of transduction with small volumes of the applied viral vector suspension. In addition to increased titres, concentrated lentiviral vector preparations benefit from concomitant purification of viral vector particles from transduction inhibitors, which are commonly present in the cell culture supernatants. Ultracentrifugation is a traditional method to concentrate lentiviral vector virions, however it is often accompanied by the loss of a substantial fraction of the active lentiviral vector particles. The particles are, in part, inactivated because of the contact between the vector pellet and air. To make the pellet more compact, ultracentrifugation is performed in swingout bucket rotors and conical-bottom tubes supplemented with appropriate adaptors. Centrifugal force can also cause deterioration of sedimenting vector particles, so a bottom layer of dense sucrose solution is recommended as a "cushion" for better survival of the vector virions. The cushion is also important because a considerable portion of the lentiviral vector preparation can be lost due to incomplete wash-off of the viral vector pellet from the tube or its poor re-suspension. VSV-G pseudotyped lentiviral vectors have relatively short half-life of $8-9 \mathrm{hrs}$ at $37{ }^{\circ} \mathrm{C}$ and are better stored at $-80{ }^{\circ} \mathrm{C}$.

Similarly to other virions, lentiviral vector particles can be precipitated using "molecular crowding" agents such as polyethylene glycol (PEG), which can soak up water molecules and create micro-pockets with an extra-high virion concentration. Thus, precipitation of virions can be achieved by a proprietary reagent "Lenti- $\mathrm{X}^{\mathrm{TM}}$ concentrator" supplied by Clontech-Takara. Precipitated lentiviral vector particles can be pelleted by low-speed centrifugation and re-suspended in a smaller volume. However, some non-lentiviral material from cell culture supernatants tends to co-precipitate with virions complicating the re-suspension step. Therefore, additional viral vector purification is required, which is often performed by ion exchange chromatography. Lentiviral vector particles are eluted from the ion exchange column in a high salt solution, which can be toxic to cells. Therefore, the medium for lentiviral vector particles should be exchanged to the desired one using dialysis, gel filtration or ultrafiltration. Ultrafiltration also provides a means for additional concentration of the viral vector.

A completely different strategy for the lentiviral vector concentration is to bind the vector particles to a coated plastic surface, which can then be used as a substrate for the cells to be infected. A recombinant derivative of human fibronectin called RetroNectin ${ }^{\mathrm{TM}}$, which was 
originally used for surface immobilisation of amphotropic retroviral vectors, can be used to trammel VSV-G pseudotyped lentiviral vectors on a plastic surface (Clontech-Takara). Lentiviral vector virions can also be concentrated, including directly at the recipient cell surfaces, after conjugation to paramagnetic particles and attraction by magnet.

Receptors for VSV-G protein are ubiquitous, but still poorly defined. Various types of cells are transduced by VSV-G-pseudotyped lentiviral vectors with different efficiency. A higher efficiency of transduction can often be achieved by a better match of the recipient cells' type and the type of the envelope protein used to pseudotype lentiviral vector particles. Lentiviral transduction is normally dramatically enhanced by polycations, such as hexadimethrine bromide (Polybrene), presumably because they modify the electrostatic interaction between the cells and the vector particles. The polycations' transduction enhancer activity is highly cell specific. Alternative polycations, such as positively charged lipid dioctadecylamidoglycylspermine (DOGS), can be more effective than Polybrene for specific cell populations (Tolmachov, Ma et al. 2006). Transduction efficiency can be further increased by a very unusual and poorly understood method called "spinfection". The recipient cells are overlaid by a viral vector suspension and centrifuged at low speed for 45 min or more. In most circumstances, such a centrifugation step results in a distinct increase in the transduction efficiency, perhaps because of the changes in the trajectories of vectorladen endosomal vesicles due to the centrifugal force.

Lentiviral vectors are able to transduce non-dividing cells. However, higher efficiencies of transduction are achieved with dividing cells, indicating that the nuclear envelope still constitutes a difficult barrier to negotiate for the entering lentiviral vector particles. Therefore, growth factors that stimulate cell division can be applied to increase the efficiency of the lentiviral transduction.

In addition to the measurement of the transduction activity, a lentiviral vector titre can be estimated using physical methods such as electron microscopy or enzyme-linked immunosorbent assay (ELISA) for capsid protein (such as "p24-antigen" of HIV-1). The titre of viral vector particles can be inferred from the concentration of genomic RNA determined by quantitative (real time) PCR. As the proportion of defective viral vector particles can vary depending on the procedures used to isolate and to concentrate the vector, such projections should rely on individually built calibration plots relating the physical titre and the transduction-based titre for a specific set of the viral vector production protocols.

\section{Conclusion}

Lentiviral gene vectors are generated by the packaging of the RNA transcribed in mammalian cells from vector backbone plasmids propagated in bacteria. Such plasmids contain bacterial and eukaryotic sets of elements. The bacterial set is comprised of a plasmid origin of replication, a bacterial selection marker, an optional partition region for stable maintenance in bacteria and plasmid DNA manipulation prop-ups like multiple cloning sites and site-specific recombination sites. The eukaryotic set consists of the lentiviral cisacting determinants for nuclear export of vector genomes, their packaging, entry into the target nuclei and chromosomal integration, plus a cargo gene or multiple cargo genes with their regulatory elements, an optional marker gene and the elements required for stable maintenance of transgenes in human cells. The bacterial set of elements is lost after vector genome RNA is produced and packaged into lentiviral transducing particles. Different cargo genes can be delivered with lentiviral vectors including genes for proteins, shRNA 
and miRNA. Currently lentiviral vectors are packaged in mammalian cells only (Lesch, Laitinen et al. 2011). The development of straightforward lentiviral vector production systems in easily cultured insect cells and yeast would be desirable (Tolmachov 2006).

\section{References}

Al-Allaf, F. A., C. Coutelle, et al. (2010). "LDLR-Gene therapy for familial hypercholesterolaemia: problems, progress, and perspectives." Int Arch Med 3: 36.

Apolonia, L., S. N. Waddington, et al. (2007). "Stable gene transfer to muscle using nonintegrating lentiviral vectors." Mol Ther 15(11): 1947-54.

Argyros, O., S. P. Wong, et al. (2011). "Development of S/MAR minicircles for enhanced and persistent transgene expression in the mouse liver." J Mol Med 89(5): 515-29.

Atasoy, D., Y. Aponte, et al. (2008). "A FLEX switch targets Channelrhodopsin-2 to multiple cell types for imaging and long-range circuit mapping." J Neurosci 28(28): 7025-30.

Attal, J., M. C. Theron, et al. (1999). "Effect of intercistronic length on internal ribosome entry site (IRES) efficiency in bicistronic mRNA." Gene Expr 8(5-6): 299-309.

Bagchi, B., M. Kumar, et al. (2006). "CMV promotor activity during ES cell differentiation: potential insight into embryonic stem cell differentiation." Cell Biol Int 30(6): 50513.

Bayer, M., B. Kantor, et al. (2008). "A large U3 deletion causes increased in vivo expression from a nonintegrating lentiviral vector." Mol Ther 16(12): 1968-76.

Burgess-Brown, N. A., S. Sharma, et al. (2008). "Codon optimization can improve expression of human genes in Escherichia coli: A multi-gene study." Protein Expr Purif 59(1): 94-102.

Dalba, C., B. Bellier, et al. (2007). "Replication-competent vectors and empty virus-like particles: new retroviral vector designs for cancer gene therapy or vaccines." Mol Ther 15(3): 457-66.

Deisseroth, K. (2011). "Optogenetics." Nat Methods 8(1): 26-9.

Dull, T., R. Zufferey, et al. (1998). "A third-generation lentivirus vector with a conditional packaging system." J Virol 72(11): 8463-71.

Ferris, A. L., X. Wu, et al. (2010). "Lens epithelium-derived growth factor fusion proteins redirect HIV-1 DNA integration." Proc Natl Acad Sci U S A 107(7): 3135-40.

Fux, C., D. Langer, et al. (2004). "New-generation multicistronic expression platform: pTRIDENT vectors containing size-optimized IRES elements enable homing endonuclease-based cistron swapping into lentiviral expression vectors." Biotechnol Bioeng 86(2): 174-87.

Gallo, P., S. Grimaldi, et al. (2008). "A lentiviral vector with a short troponin-I promoter for tracking cardiomyocyte differentiation of human embryonic stem cells." Gene Ther 15(3): 161-70.

Geraerts, M., S. Willems, et al. (2006). "Comparison of lentiviral vector titration methods." BMC Biotechnol 6: 34.

Goverdhana, S., M. Puntel, et al. (2005). "Regulatable gene expression systems for gene therapy applications: progress and future challenges." Mol Ther 12(2): 189-211. 
Grandchamp, N., D. Henriot, et al. (2011). "Influence of insulators on transgene expression from integrating and non-integrating lentiviral vectors." Genet Vaccines Ther 9(1): 1 .

Hager, S., F. M. Frame, et al. (2008). "An internal polyadenylation signal substantially increases expression levels of lentivirus-delivered transgenes but has the potential to reduce viral titer in a promoter-dependent manner." Hum Gene Ther 19(8): 840-50.

Hiller, M. and M. Platzer (2008). "Widespread and subtle: alternative splicing at shortdistance tandem sites." Trends Genet 24(5): 246-55.

Humme, S., G. Reisbach, et al. (2003). "The EBV nuclear antigen 1 (EBNA1) enhances B cell immortalization several thousandfold." Proc Natl Acad Sci U S A 100(19): 1098994.

Kita-Matsuo, H., M. Barcova, et al. (2009). "Lentiviral vectors and protocols for creation of stable hESC lines for fluorescent tracking and drug resistance selection of cardiomyocytes." PLoS One 4(4): e5046.

Kuate, S., D. Stefanou, et al. (2004). "Production of lentiviral vectors by transient expression of minimal packaging genes from recombinant adenoviruses." J Gene Med 6(11): 1197-205.

Kustikova, O. S., A. Wahlers, et al. (2003). "Dose finding with retroviral vectors: correlation of retroviral vector copy numbers in single cells with gene transfer efficiency in a cell population." Blood 102(12): 3934-7.

Le Hir, H., A. Nott, et al. (2003). "How introns influence and enhance eukaryotic gene expression." Trends Biochem Sci 28(4): 215-20.

Lesch, H. P., A. Laitinen, et al. (2011). "Production and purification of lentiviral vectors generated in 293T suspension cells with baculoviral vectors." Gene Ther, in press.

Lombardo, A., P. Genovese, et al. (2007). "Gene editing in human stem cells using zinc finger nucleases and integrase-defective lentiviral vector delivery." Nat Biotechnol 25(11): 1298-306.

Luke, J., A. E. Carnes, et al. (2009). "Improved antibiotic-free DNA vaccine vectors utilizing a novel RNA based plasmid selection system." Vaccine 27(46): 6454-9.

Maier, P., C. von Kalle, et al. (2010). "Retroviral vectors for gene therapy." Future Microbiol 5: 1507-23.

Moldt, B., N. H. Staunstrup, et al. (2008). "Genomic insertion of lentiviral DNA circles directed by the yeast Flp recombinase." BMC Biotechnol 8: 60.

Nightingale, S. J., R. P. Hollis, et al. (2006). "Transient gene expression by nonintegrating lentiviral vectors." Mol Ther 13(6): 1121-32.

Philippe, S., C. Sarkis, et al. (2006). "Lentiviral vectors with a defective integrase allow efficient and sustained transgene expression in vitro and in vivo." Proc Natl Acad Sci U S A 103(47): 17684-9.

Philpott, N. J. and A. J. Thrasher (2007). "Use of nonintegrating lentiviral vectors for gene therapy." Hum Gene Ther 18(6): 483-9.

Rahim, A. A., A. M. Wong, et al. (2009). "Efficient gene delivery to the adult and fetal CNS using pseudotyped non-integrating lentiviral vectors." Gene Ther 16(4): 509-20. 
Reumers, V., C. M. Deroose, et al. (2008). "Noninvasive and quantitative monitoring of adult neuronal stem cell migration in mouse brain using bioluminescence imaging." Stem Cells 26(9): 2382-90.

Riviere, L., J. L. Darlix, et al. (2010). "Analysis of the viral elements required in the nuclear import of HIV-1 DNA." J Virol 84(2): 729-39.

Rubinson, D. A., C. P. Dillon, et al. (2003). "A lentivirus-based system to functionally silence genes in primary mammalian cells, stem cells and transgenic mice by RNA interference." Nat Genet 33(3): 401-6.

Sarkis, C., S. Philippe, et al. (2008). "Non-integrating lentiviral vectors." Curr Gene Ther 8(6): 430-7.

Schambach, A., J. Bohne, et al. (2006). "Woodchuck hepatitis virus post-transcriptional regulatory element deleted from $X$ protein and promoter sequences enhances retroviral vector titer and expression." Gene Ther 13(7): 641-5.

Schoenenberger, P., D. Gerosa, et al. (2009). "Temporal control of immediate early gene induction by light." PLoS One 4(12): e8185.

Sektas, M., N. Hasan, et al. (2001). "Expression plasmid with a very tight two-step control: Int/att-mediated gene inversion with respect to the stationary promoter." Gene 267(2): 213-20.

Silva, J. M., M. Z. Li, et al. (2005). "Second-generation shRNA libraries covering the mouse and human genomes." Nat Genet 37(11): 1281-8.

So, P. W., S. Hotee, et al. (2005). "Generic method for imaging transgene expression." Magn Reson Med 54(1): 218-21.

Sohal, V. S., F. Zhang, et al. (2009). "Parvalbumin neurons and gamma rhythms enhance cortical circuit performance." Nature 459(7247): 698-702.

Soifer, H., C. Higo, et al. (2002). "A novel, helper-dependent, adenovirus-retrovirus hybrid vector: stable transduction by a two-stage mechanism." Mol Ther 5(5 Pt 1): 599608.

Srinivasakumar, N. (2001). "HIV-1 vector systems." Somat Cell Mol Genet 26(1-6): 51-81.

Tolmachov, O. (2006). "A heterologous system for assembly of retroviral gene vectors: intracellular budding in yeast?" Med Hypotheses 67(4): 807-9.

Tolmachov, O. (2009). "Designing plasmid vectors." Methods Mol Biol 542: 117-29.

Tolmachov, O., Y. L. Ma, et al. (2006). "Overexpression of connexin 43 using a retroviral vector improves electrical coupling of skeletal myoblasts with cardiac myocytes in vitro." BMC Cardiovasc Disord 6: 25.

Vilaboa, N. and R. Voellmy (2006). "Regulatable gene expression systems for gene therapy." Curr Gene Ther 6(4): 421-38.

Wanisch, K. and R. J. Yanez-Munoz (2009). "Integration-deficient lentiviral vectors: a slow coming of age." Mol Ther 17(8): 1316-32.

Weber, W., M. Rimann, et al. (2004). "Gas-inducible transgene expression in mammalian cells and mice." Nat Biotechnol 22(11): 1440-4.

Wong, S. P., O. Argyros, et al. (2009). "Strategies for the episomal modification of cells." Curr Opin Mol Ther 11(4): 433-41.

Wong, S. P., O. Argyros, et al. (2011). "Non-viral S/MAR vectors replicate episomally in vivo when provided with a selective advantage." Gene Ther 18(1): 82-7. 
Yanez-Munoz, R. J., K. S. Balaggan, et al. (2006). "Effective gene therapy with nonintegrating lentiviral vectors." Nat Med 12(3): 348-53.

Zeeberg, B. (2002). "Shannon information theoretic computation of synonymous codon usage biases in coding regions of human and mouse genomes." Genome Res 12(6): 944-55.

Zhu, X., I. Chung, et al. (2001). "Coexpression of normal and mutated CD40 ligand with deletion of a putative RNA lariat branchpoint sequence in X-linked hyper-IgM syndrome." Clin Immunol 99(3): 334-9. 


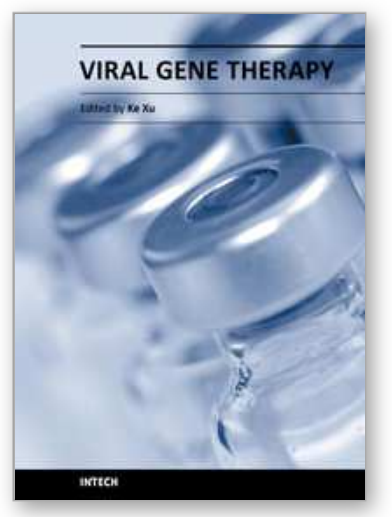

\author{
Viral Gene Therapy \\ Edited by Dr. Ke Xu
}

ISBN 978-953-307-539-6

Hard cover, 450 pages

Publisher InTech

Published online 20, July, 2011

Published in print edition July, 2011

The development of technologies that allow targeting of specific cells has progressed substantially in recent years for several types of vectors, particularly viral vectors, which have been used in $70 \%$ of gene therapy clinical trials. Particular viruses have been selected as gene delivery vehicles because of their capacities to carry foreign genes and their ability to efficiently deliver these genes associated with efficient gene expression. This book is designed to present the most recent advances in viral gene therapy

\title{
How to reference
}

In order to correctly reference this scholarly work, feel free to copy and paste the following:

Oleg Tolmachov, Tanya Tolmachova and Faisal A. Al-Allaf (2011). Designing Lentiviral Gene Vectors, Viral Gene Therapy, Dr. Ke Xu (Ed.), ISBN: 978-953-307-539-6, InTech, Available from: http://www.intechopen.com/books/viral-gene-therapy/designing-lentiviral-gene-vectors

\section{INTECH}

open science | open minds

\author{
InTech Europe \\ University Campus STeP Ri \\ Slavka Krautzeka 83/A \\ 51000 Rijeka, Croatia \\ Phone: +385 (51) 770447 \\ Fax: +385 (51) 686166 \\ www.intechopen.com
}

\author{
InTech China \\ Unit 405, Office Block, Hotel Equatorial Shanghai \\ No.65, Yan An Road (West), Shanghai, 200040, China \\ 中国上海市延安西路65号上海国际贵都大饭店办公楼 405 单元 \\ Phone: +86-21-62489820 \\ Fax: +86-21-62489821
}


(C) 2011 The Author(s). Licensee IntechOpen. This chapter is distributed under the terms of the Creative Commons Attribution-NonCommercialShareAlike-3.0 License, which permits use, distribution and reproduction for non-commercial purposes, provided the original is properly cited and derivative works building on this content are distributed under the same license. 